\title{
Spectrum of congenital heart diseases in an African population: A necropsy study
}

\author{
MO Thomas, Olugbenga Olusoji, Nicholas Awolola*
}

Cardiothoracic Surgery Unit, Department of Anatomic and Molecular Pathology, Lagos University Teaching Hospital/College of Medicine of University of Lagos, Idi-araba, Lagos, Nigeria

Email: oluwafemithomas@yahoo.com

Received 21 November 2012; revised 25 December 2012; accepted 31 December 2012

\begin{abstract}
Background: The spectrum and incidence of congenital heart diseases amongst African population are not well defined in literature. There is the need to further elucidate the spectrum and epidemiology of congenital heart diseases amongst African. Aim: This study was conducted to highlight the spectrum and occurrence of congenital heart diseases in an African population. Methodology: All paediatric patients who were autopsied over a 98-month period were checked for occurrence of congenital heart diseases. Attempts were made to ascertain the primary disease and causes of death. We noted their bio-data, ages, sexes and causes of death. The results were analysed with SPSS version 17. The results formed the basis for discussion and recommendations. Results: There were 135 cardiac related deaths and 36 of them were in paediatric age brackets. The mean age was $4.7+1.08$ months with a standard deviation of 6.27. M:F ratio was 1:1.1. The commonest anomaly was ventricular septal defect. There was a weak association with other congenital anomalies. The commonest cause of death was bronchopneumonia. Discussion and Recommendation: The disease pattern showed reasonable similarity with reports from other parts of the world. However, there is need for development of capacity for prenatal diagnosis of congenital heart diseases in Africa. This will widen the net of diagnosis and improve accuracy of incidence studies in Africa.
\end{abstract}

Keywords: Congenital Heart Diseases Necropsy

\section{INTRODUCTION}

Paediatric cardiac diseases are either congenital or acquired and they exhibit spectacular spectrum. It is common knowledge that some children with congenitally malformed hearts survive into adulthood: this is the same pattern with some acquired heart diseases. This situation

\footnotetext{
"Corresponding author.
}

often leads to some continuation of childhood cardiac disease pattern into adulthood in some patients. From the early 1940s through the 1950s, it was a popular belief throughout the world that cardiac diseases were rare among Africans [1].

Ever since the establishment of Ibadan cardiac registry in 1964, all types of cardiac diseases, including those that are amenable to surgery were revealed in Nigeria [2]. Earlier reports have shown that little was known about the incidence and types of congenital heart diseases (CHD) in African children.

In a study of 4220 consecutive births in Ibadan, South West Nigeria, the derived incidence of CHD was 3.5 per 1000 births [2]. The overall incidence of congenital malformations was 3\% and CHD formed one-eight of the total malformations. The commonest isolated CHD in Nigeria is ventricular septal defect (VSD) which constitutes $27 \%$ of the cases followed by patent ductus arteriosus (PDA) 18\%, atrial septal defect (ASD) (14.2\%) and pulmonary stenosis (12.3\%) [3].

The commonest multiple defects in Nigeria is Tetralogy of Fallot (TOF) and these occur in $12.3 \%$ of series. Sex incidence is equal, thus keeping with findings elsewhere. With above findings Antia (1974) concluded that the pattern of CHD in Nigeria is the same as it is elsewhere in the world [1-5].

It is thought that most congenital cardiovascular malformations are probably a result of complex interactions between genetic and environmental factors [4]. Even at that it is also believed that racial difference may not play any significant role in incidence of CHDs. Associated anomalies tend to occur commonly with CHDs [1-5]. A number of studies have attempted to define associations between specific cardiac defects and non-cardiac malformations [6,7].

Among these associations, the well established ones are the association between Down Syndrome and endocardial cushion defects or ventricular septal defects (VSD), that between agenesis of spleen and conotruncal anomalies and between limb reductions and septal defects in 
patients with autosomal dominant Holt-Oram syndrome $[8,9]$.

Congenital malformations of the heart are known leading causes of death in newborn [5]. Contemporary population-based epidemiologic data using echocardiography to detect valve diseases suggest that the prevalence of valve diseases in Africa is much higher than previously thought; meaning that the estimated mortality, morbidity and socio-economic burden they cause could also be much higher than currently appreciated [10].

Hospital-based studies and causes of death studies across Africa are all consistent and show that rheumatic heart disease is the main acquired cause of cardiac morbidity and mortality in children and young adults [11]. Heart failure as a complication is the predominant problem, followed by other complications such as native valve infective endocarditis, systemic embolisation, pulmonary hypertension, cardiac arrhythmias or complications related to valve surgery [11].

In all, previous studies of heart diseases in children have not conclusively established autopsy pattern of heart diseases in this part of the world. This research was conducted to further unravel the pattern of heart diseases in children as found in autopsy studies.

\section{PATIENTS AND METHODS}

This research is a necropsy study of congenital heart diseases (CHD) patients. It was a hospital-based study spanning a period of 98 months. During this period, the records of all postmortem cases were compiled and the cardiac cases were isolated. Out of these, the congenital heart diseases, causes of death were spotted for further analysis. We noted their biodata and other demographic data-including their ages and sexes. We noted the structural defects, and postmortem findings of causes of death.

The data were entered into and analysed with SPSS version 17. The output formed the basis for the information that was used in the section for discussion. We looked at diseases specific demographic data and causes of death. Inferences therefrom were the bases for the subsequent recommendations under discussion.

\section{RESULTS}

The study period covered 98 months during which 789 autopsies were carried out. There were 135 cardiac related deaths constituting $17.1 \%$ of all cases. Out of those, there were 36 paediatric cases. Therefore the paediatric cases constituted $26.7 \%$ of cardiac related death. Average postmortem rate in this group was 0.4 per month.

\subsection{Age and Sex Distribution of Cases}

There were 36 patients $(n=36)-17$ males $(47.2 \%)$ and
19 (52.8\%) females giving a $\mathrm{M}: \mathrm{F}$ ratio of $1: 1.3$. The age range was 32.0 months, (minimum was 22 hours and maximum was 32 months). The mean age was $4.7 \pm 1.08$ months.

The median age was 3 months and the mode was 4 months. Measurement of age dispersion showed standard deviation of 6.27. The distribution was positively skewed (standard Error of Skewness was 0.403). It was also leptokurtic with a standard error of kurtosis of 0.788 .

\subsection{Primary Diseases and Sex Distribution}

There were 8 cases of ventricular septal defects constituting $22.2 \%$ of the lot. This was followed by atrial septal defects (13.9\%), and 11.1\% were tetralogy of Fallot.

Patent ductus arteriosus and transposition of great arteries (TGA) were 3 each, constituting $8.3 \%$ of cases. The overall M:F ratio was 1:1.3. Further evaluation showed M:F ratio of 1:3 for ventricular septal defects, 1:1 for atrial septal defects, 1:2 for patent ductus arteriosus and transposition, 1:1 for tetralogy of Fallot. All the atrio-ventricular septal defects (AVSD) were males.

\subsection{Other Anomalies}

We saw one case each of congenital diaphragmatic hernia, Down's syndrome, pulmonary hypoplasia, renal agenesis, situs inversus and uniatrium. This generated a Pearson Chi-square significance 0.381 and a likelihood ratio of 82.634. The $\mathrm{M}: \mathrm{F}$ ratio for associated anomalies was $1: 1.2$.

\subsection{Causes of Death}

Bronchopneumonia which occurred in $25.0 \%$ of cases, was the commonest cause of death. Congestive cardiac failure and lung collapse were found in 6 patients (16.7\%) each. Up to $50.0 \%$ of deaths were recorded by two months of age. Figure 1 shows the ages at death (in months) and the frequency of deaths.

Frequency distribution of primary diseases showed that by the age of 2 months, 6 ventricular septal defect deaths had been recorded while 2 deaths occurred in the atrial septal defect group within the same period.

The postmortem findings (causes of death) in all categories are illustrated in Figure 2. By the age of 2 months, the commonest cause of death was lung collapse as seen in $33.3 \%$ of cases within that age bracket.

Figure 3 shows occurrence of death by date in different periods of the year. Figure 4 shows the stem-and-leaf plot of causes of death by age in months.

\section{DISCUSSION}

Congenital heart diseases (CHD) are one of the important 
groups of birth defects and they contribute significantly to infant mortality [5].

The total prevalence of congenital heart diseases has been estimated at 3.9 - 11.9 per 1000 live-born infants by different authors. These reported rates differ significantly because of variations in diagnostic and registration criteria and the percentage of prenatally diagnosed cases are included in some of the studies $[12,13]$.

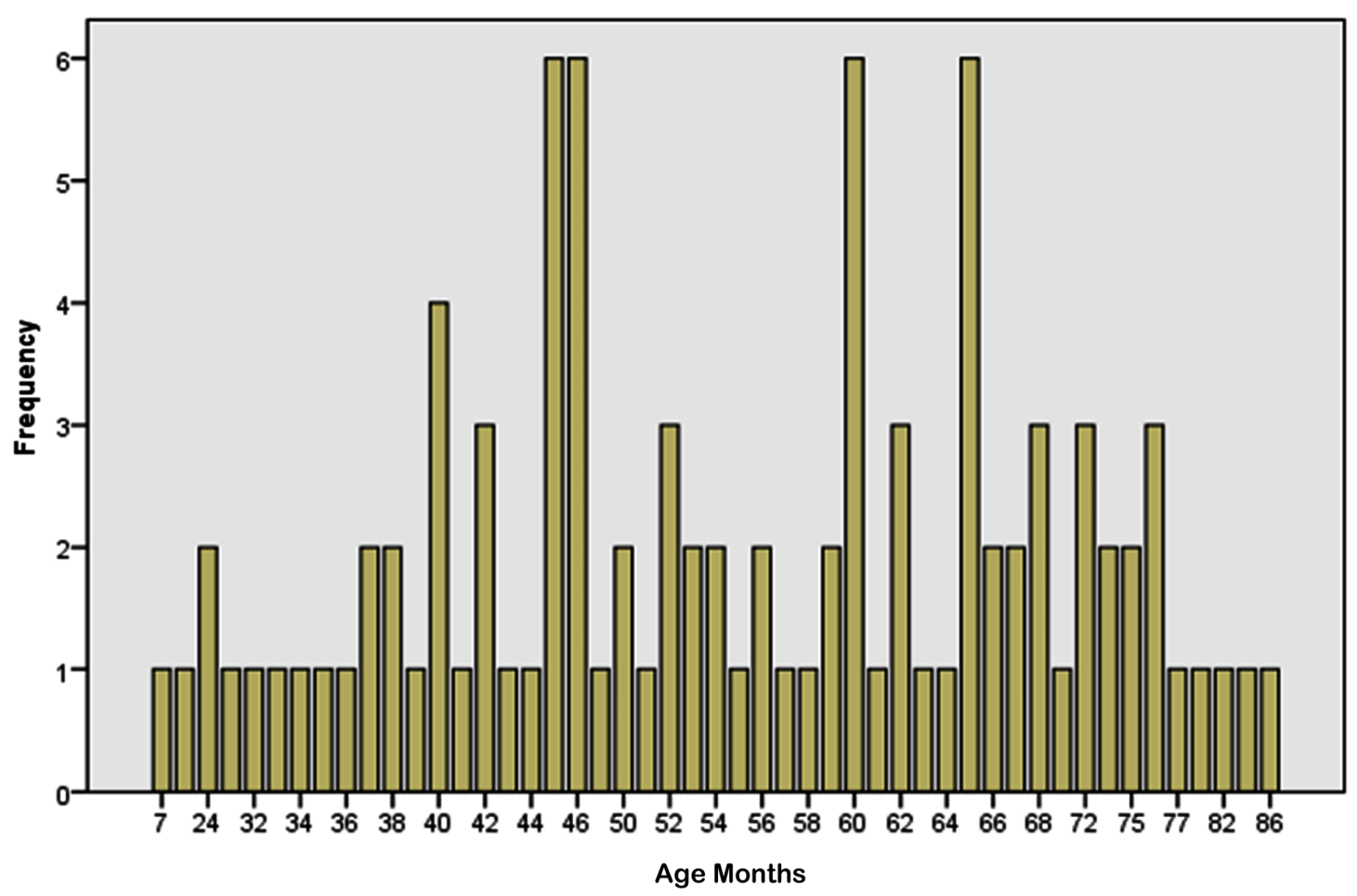

Figure 1. Age distribution of all death.

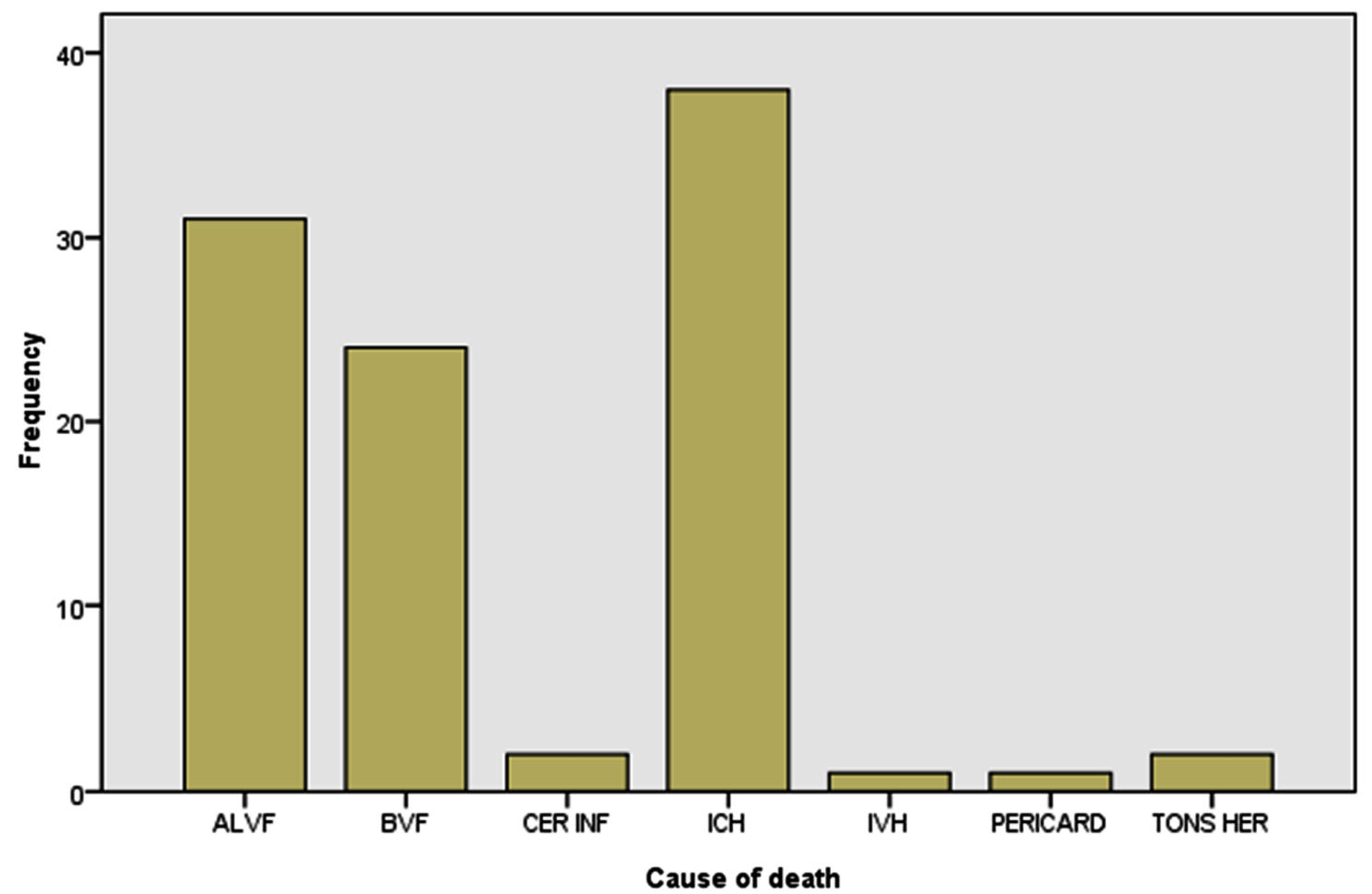

Figure 2. Bar chart of causes of death. 


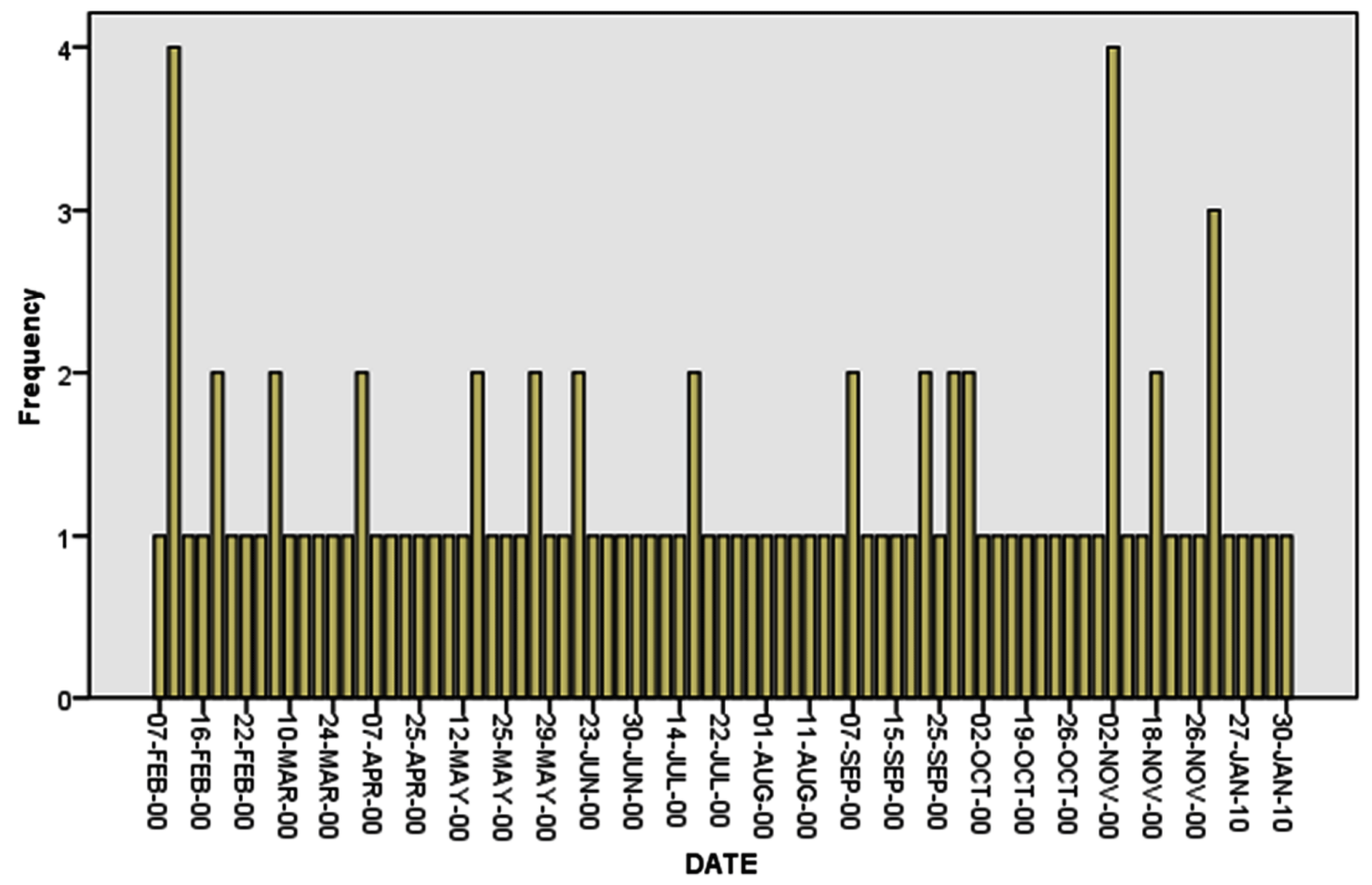

Figure 3. Occurrence of deaths by date.

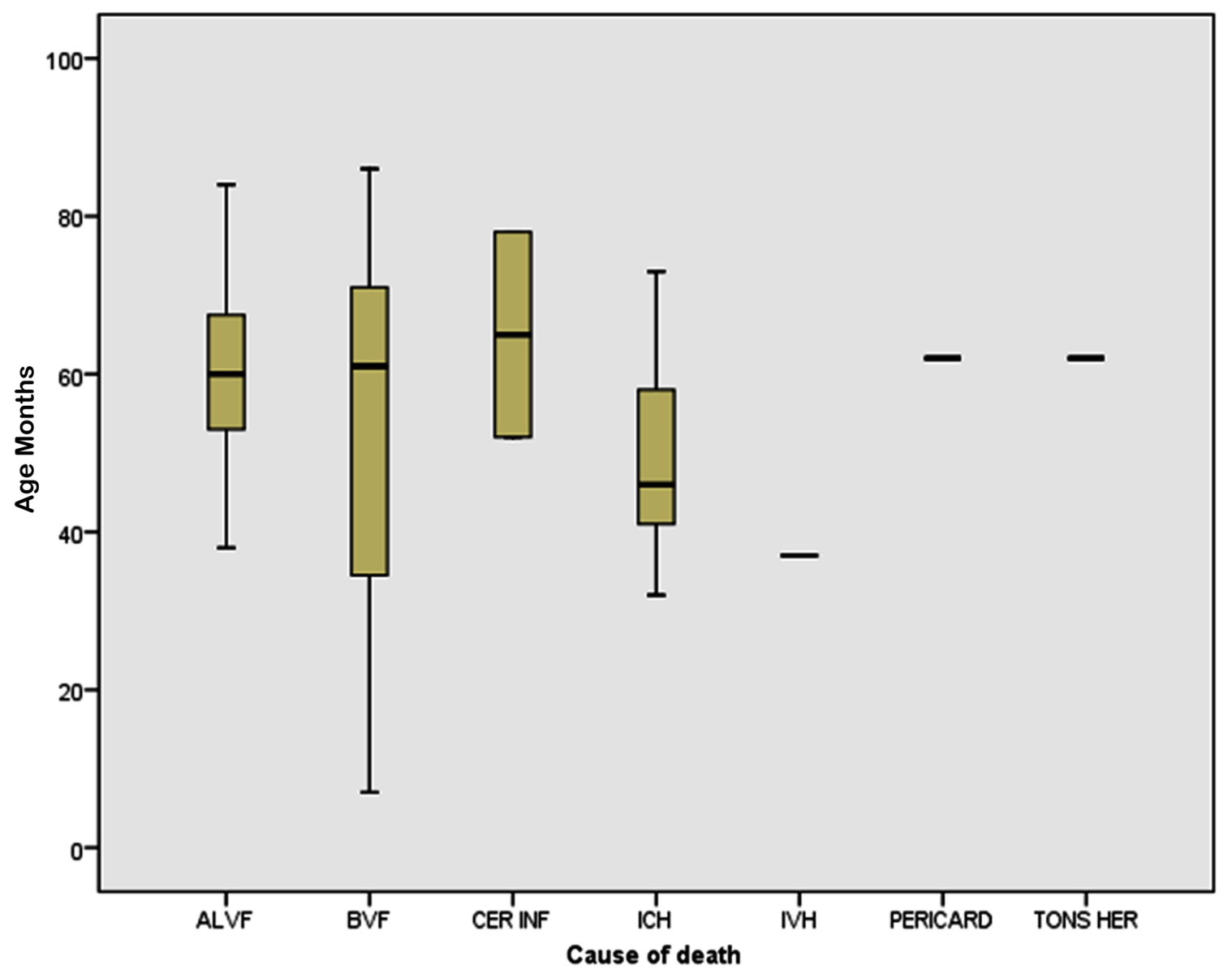

Figure 4. Stem-and-leaf plot of causes of death. 
In Nigeria, the incidence of congenital heart diseases is 3.5 per 1000 births [2]. The overall incidence of congenital malformation is 3 percent and CHDs constitute one-eight of all malformations [2].

In this necropsy study, $17.1 \%$ of deaths were cardiac related and $26.7 \%$ of the cardiac deaths were due to CHD. Therefore, there is no uniform platform for comparison of CHD with the total number of congenital malformations in this situation and not all cases of CHDs would have died during the study period. Definitely, some would have lived into adulthood.

Overall, 4.6\% of autopsied bodies had CHDs. This is lower than the 9.1\% rate in the Turkish [5]. The M:F ratio of 1:1.3 in our series is similar to the occurrence in many regions of the world. Ventricular septal defect was the commonest as it was found in $22.2 \%$ of the cases. This was followed by atrial septal defects (ASD) (13.9\%) and tetralogy of Fallot (11.1\%).

In a similar necropsy study conducted in Turkey, Gucer and his colleagues [5] found ventricular septal defect as the most common CHD (15.3\%), followed by ASD and transposition of great arteries (TGA). All the TGAs were of d-type. In our series, TGA and patent ductus arteriosus (PDA) took the $4^{\text {th }}$ position constituteing $8.3 \%$ each.

The overall M:F ratio was 1:1.3. We saw a bit of variation in diseases-specific male to female ratios. It was 1:3 for VSDs, 1:1 for ASDs 1:2 for PDAs and TDAs. All the atrio-ventricular septal defects (AVSD) were males. Association with other anomalies showed a weak relationship. We saw one case each of Down's syndrome, pulmonary hypoplasia, renal agenesis and situs inversus. The likelihood ratio was 82.634.

Overall, the M:F ratio for associated anomalies was 1:1.2. This is also very close to the overall M:F ratio despite the weak likelihood ratio. The main causes of death were bronchopneumonia (25.0\%) and congestive cardiac failure (16.7\%).

In a similar study conducted in Ibadan South Western Nigeria in $1974,61.7 \%$ of the patents died by the sixth month. This is at variance with the findings in our study where the mean age was $4.7 \pm 1.08$ months. The age distribution showed a semblance of normality because the mean, mode and median were very close. The dispersion showed a standard deviation of 6.27.

It is therefore worthy of note that most patients died before age one. These could be explained from poor access to medical attention, poverty and ignorance. All these problems constitute the bulk of unresolved issues in the developing world.

It is therefore important that for any meaningful program to be recorded in treatment of CHD, there is need for appropriate policies for early detection and prompt treatment. These will reduce the incidence of attendant complication which will make the overall outlook to be better.

Prenatal diagnosis is another important aspect of care that is mostly neglected in the developing world. It is expected that if this is made available, more severe anomalies would be detected prematurely and care would begin early—even before reaching the maternity ward.

It is recommended that concerted effort should be made to develop adequate diagnostic and treatment programs aimed at improving the outlook of CHDs in the developing world.

\section{REFERENCES}

[1] Eze, J.C. and Ezemba, N. (2007) Open-heart surgery in Nigeria: Indications and challenges. Texas Heart Institute Journal, 34, 8-10.

[2] Gupta, B. and Antia, A.U. (1967) Incidence of congenital heart disease in Nigerian children. British Heart Journal, 29, 906-909. doi:10.1136/hrt.29.6.906

[3] Antia, A.U. (1974) Congenital heart diseases in Nigeria: Clinical and necropsy study of 260 cases. Archives of Diseases in Childhood, 49, 36-39. doi:10.1136/adc.49.1.36

[4] Maron, B.J., Applefeld, J.M. and Krovetz, L.J. (1973) Racial frequencies in congenital heart disease. Circulation, 47, 359-361. doi:10.1161/01.CIR.47.2.359

[5] Gucer, S., Ince, T., Kale, G., Akwren, Z., Talin, S.O.B. and Caglar, M. (2005) Non cardiac malformations in congenital heart disease: A retrospective analysis of 305 paediatric autopsies. The Turkish Journal of Pediatrics, 47, 159-166.

[6] Pinar, H. (2004) Postmortem findings in term neonates. Seminars in Neonatology, 9, 289-302. doi:10.1016/j.siny.2003.11.003

[7] Greenwood, R.D., Rosenthal, A., Fyler, D. and Nadas, A.S. (1975) Extracardiac abnormalities in infants with congenital heart diseases. Paediatrics, 55, 485-492.

[8] Ivemark, B.I. (1955) Implications of agenesis of the spleen on the pathogenesis of conotruncus anomalies in childhood; an analysis of the heart malformations in the splenic agenesis syndrome with fourteen new cases. Acta Paediatrica, 44, 7-110. doi:10.1111/j.1651-2227.1955.tb05346.x

[9] Holt, M. and Oram, S. (1960) Familial heart disease with skeletal malformations. British Heart Journal, 22, 236242. doi:10.1136/hrt.22.2.236

[10] Nkomo, V.T. (2009) Epidemiology of valvular heart diseases in Africa. South African, 6, 12-18.

[11] Caraptis, J.R., Steer, A.C., Mulholland, E.K., et al. (2005) The global burden of group A streptococcal disease. The Lancet Infectious Diseases, 511, 685-694.

[12] Pradat, P., Franeannet, C., Harris, J.A. and Robert, E. (2003) The epidemiology of cardiovascular defects, Part 1: A study based on data from three large registries of congenital malformations. Pediatric Cardiology, 24, 195221. doi:10.1007/s00246-002-9401-6 
[13] Tennstedt, C., Chaoul, R., Komer, H. and Dietei, M. (1999) Spectrum of congenital heart defects and extracardiac malformations associated with chromosome ab- normalities: Result of seven year necropsy study. Heart, 82, 34-39. 